\title{
Digital Reflection-Type Phase Shifter Based on a Ferroelectric Planar Capacitor
}

\author{
V. Sherman, K. Astafiev, N. Setter, A. Tagantsev, O. Vendik, Member, IEEE, I. Vendik, Member, IEEE, \\ S. Hoffmann-Eifert, U. Böttger, and R. Waser
}

\begin{abstract}
Novel design of an integrated digital refelction-type phase shifter based on a Barium Strontium Titanate thin-film capacitor is presented. Measured characteristics of the phase shifter are compared with the theoretically predicted ones.
\end{abstract}

Index Terms-Ferroelectrics, microwaves, phase shifter.

\section{INTRODUCTION}

$\mathbf{U}$ SING ferroelectric thin films for microwave phase shifter design gives the possibility to improve characteristics of the phase shifters and to decrease their cost in comparison with p-i-n diode or transistor phase shifters. Small switching time, low phase noises, and integrated technology are the main advantages of the ferroelectric phase shifters.

The ferroelectric film is characterized by a tunability $n>1$ determined as the ratio of the dielectric constants $n=\varepsilon_{1}(0) / \varepsilon_{2}\left(V_{c}\right)$ and loss factor $\tan \delta_{1,2}$ under biasing voltage $V_{1}=0$ and $V_{2}=V_{c}$ correspondingly. Having the ferroelectric film of a high quality (i.e., $n \geq 2$ and $\tan \delta \leq 0.01$ ), microwave engineers can design an integrated analog phase shifter as a section of a planar line based on the ferroelectric layer [1], [2]. Another solution is to use the ferroelectric flip-chip capacitors instead of the p-i-n diodes in refelction-type phase shifters [3]. The theory of digital refelction-type phase shifters based on lumped switching components (like pin-diodes and FETs) is well developed [4], [5] and can be adopted for the design of the phase shifters using tunable ferroelectric capacitors. This approach allows designing the phase shifter with optimized characteristics: minimal insertion loss in both states and a stable phase shift in a desired frequency band.

The refelction-type phase shifter can be converted into the transmission one by using well known methods [4].

Manuscript received April 12, 2001; revised August 2, 2001. This work was supported by the Swiss National Foundation. The review of this letter was arranged by Associate Editor Dr. Ruediger Vahldieck.

V. Sherman, K. Astafiev, N. Setter, and A. Tagantsev are with the Ecole Politechnique Federale de Lausanne, Lausanne, Switzerland.

O. Vendik and I. Vendik are with the St.-Petersburg Electrotechnical University, St. Petersburg, Russia.

S. Hoffmann-Eifert is with IFF, Research Center Jülich, Jülich, Germany.

U. Böttger is with RWTH Aachen University, Aachen, Germany.

R. Waser is with IFF, Research Center Jülich, Jülich, Germany, and with RWTH Aachen University, Aachen, Germany.

Publisher Item Identifier S 1531-1309(01)09484-3.

\section{Figure of Merit of the Ferroelectric Digital REFLECTION TYPE PHASE SHIFTER}

The main characteristic of the phase shifter is the figure of merit, which is determined as a ratio of the phase shift $\Delta \varphi$ and average loss

$$
F=\frac{\Delta \varphi(\mathrm{deg})}{\sqrt{L_{1}(\mathrm{~dB}) \cdot L_{2}(\mathrm{~dB})}}
$$

where $L_{1}$ and $L_{2}$ are the losses of the phase shifter in two different states.

The figure of merit of the phase shifter is strongly dependent on the commutation quality factor of a switchable component used in the phase shifter [6], [7] and for one-bit phase shifter can be determined as

$$
F \approx 6.6\left(\sin \frac{\Delta \varphi}{2} / \frac{\Delta \varphi}{2}\right)^{-1} \cdot \sqrt{K}
$$

where $K$ is the commutation quality factor (CQF) of the switchable component. For a ferroelectric capacitor with the capacitance $C_{1}$ and $C_{2}$ and loss tangent $\tan \delta_{1}$ and $\tan \delta_{1}$ in two different states, the CQF is

$$
K=\frac{(n-1)^{2}}{n \cdot \tan \delta_{1} \tan \delta_{2}}
$$

where $n=C_{1} / C_{2}$ is the tunability of the capacitor under the bias voltage.

Equation (2) gives the theoretical limitation of the figure of merit of the digital phase shifter. In practice, the value of the figure of merit is less, due to additional loss contribution provided by conductors and connectors.

\section{Phase Shifter Simulation and Design}

The main idea of an optimal design of refelction-type phase shifters based on switchable lumped component is formulated in [5]. The desired phase shift $\Delta \varphi$ with equal and minimal insertion loss in both states of the switchable component (ferroelectric capacitor) can be provided by using a lossless reciprocal 2-port network, which should be designed as the matching circuit for a characteristic load impedance $Z_{\text {in }}$ dependent on the tunable capacitor impedance $Z_{1,2}=r_{1,2}+i x_{1,2}$ in the two states and on the needed phase shift. For the $180^{\circ}$ phase shift, $Z_{i n}$ has been found as follows

$$
Z_{i n}^{*}=\sqrt{r_{1} r_{2}\left[\left(\frac{x_{1}-x_{2}}{r_{1}+r_{2}}\right)^{2}+1\right]}-\mathrm{i} \frac{r_{2} x_{1}+r_{1} x_{2}}{r_{1}+r_{2}} .
$$




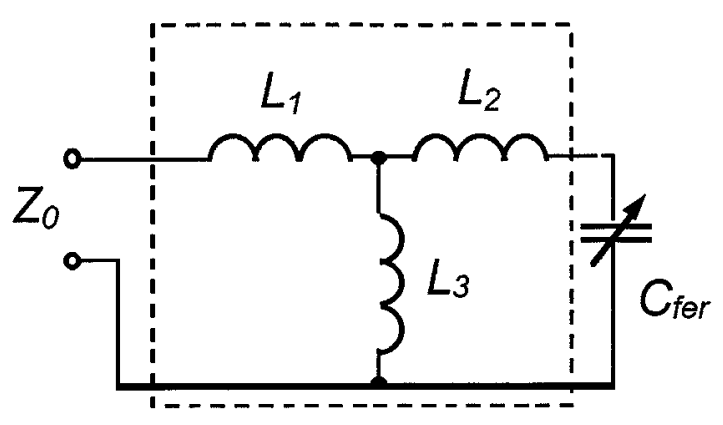

(a)

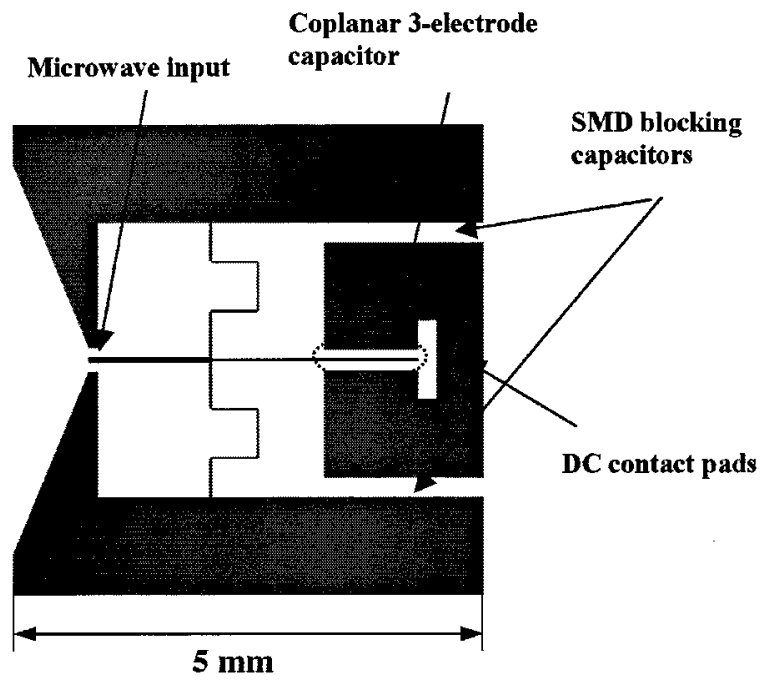

(b)

Fig. 1. (a) Equivalent electrical circuit and (b) layout of $180^{\circ}$ reflection-type integrated phase shifter based on bi-layered sapphire-BSTO substrate.

As an example, the $180^{\circ}$ reflection-type phase shifter was designed using the ferroelectric capacitor with $C_{1}=0.45 \mathrm{pF}$, $C_{2}=0.25 \mathrm{pF}, \tan \delta_{1}=\tan \delta_{2}=0.02$. The impedance $Z_{\text {in }}$ was found as $Z_{\text {in }}=15.5-\mathrm{i} 62.2 \Omega$ at $5.4 \mathrm{GHz}$. The matching network was designed as an inductance T-circuit [Fig. 1(a)].

A coplanar line structure based on the multi-layered (sapphire-BSTO film) substrate with strip lumped inductances was proposed for the one-bit phase shifter.

The phase shifter was realized on the bi-layered substrate. The $0.5 \mu \mathrm{m}$ thick BSTO film was obtained on the $0.5 \mathrm{~mm}$ thick sapphire substrate using CSD technique. A copper layer of $4 \mu \mathrm{m}$ thickness was patterned by wet photolithography. The layout of the phase shifter is presented in Fig. 1(b). The tunable capacitor was realized as a short section of a coplanar line of $100 \mu \mathrm{m}$ width with $20 \mu \mathrm{m}$ gaps. The central electrode of the capacitor is connected with the matching circuit. The coplanar input of the phase shifter was specially designed to minimize losses in the near-by-connector region. To apply dc control voltage, the ground electrode of the coplanar capacitor was connected with the common coplanar ground through nontunable SMD capacitors $(5.1 \mathrm{pF})$.

The simulation of the phase shifter was performed by HPADS software. To decrease the simulation time, the phase shifter was simulated separately for each state using different mesh setup.

The simulated performance of the phase shifter (Fig. 2) shows equal losses and the $180^{\circ}$ phase shift at $5.38 \mathrm{GHz}$. The evalu-
MS11, dB
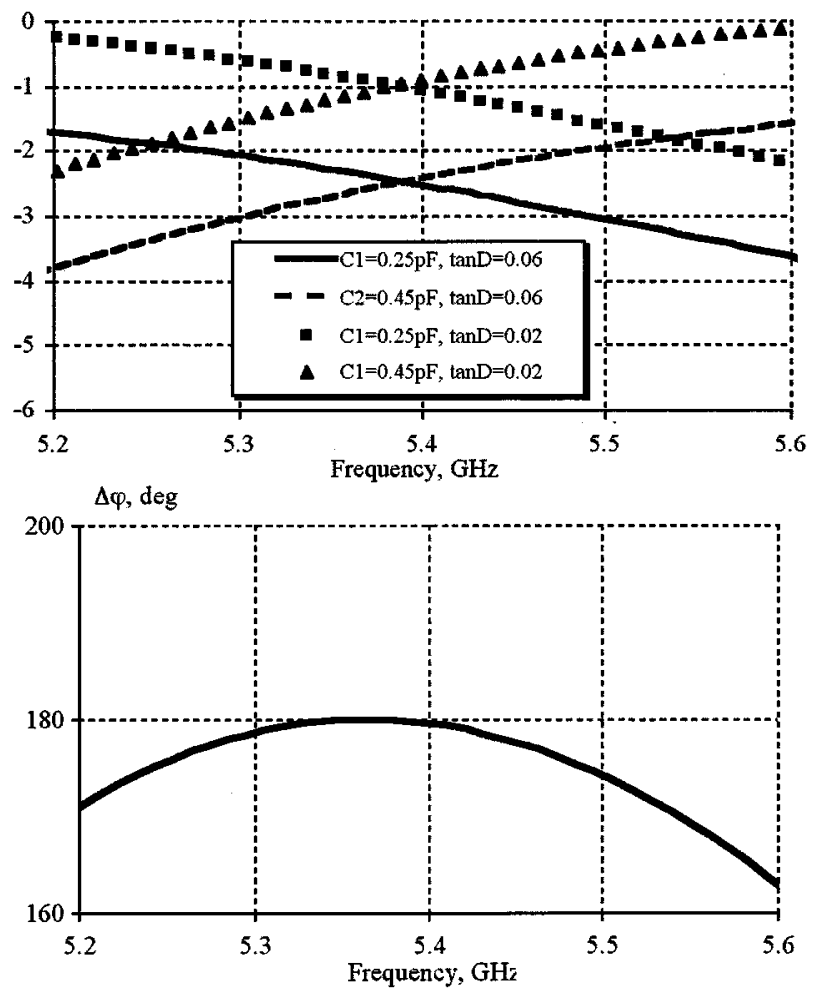

Fig. 2. Simulated performance of the phase shifter for two values of loss tangent: $\tan \delta=0.06$ and $\tan \delta=0.02$.

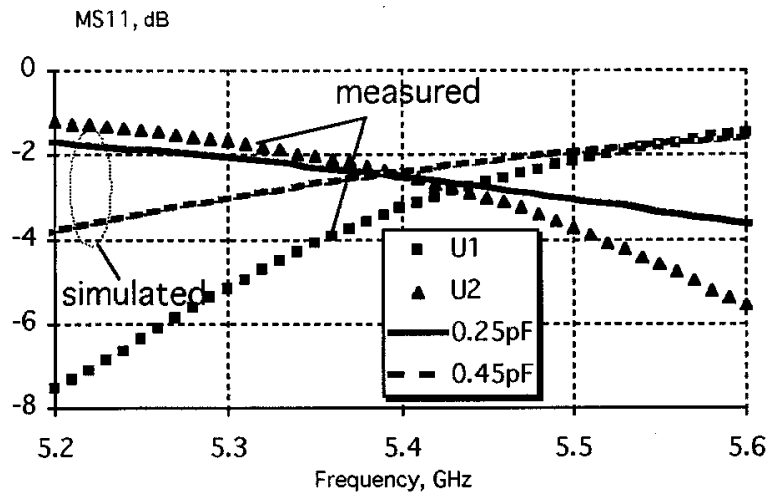

(a)

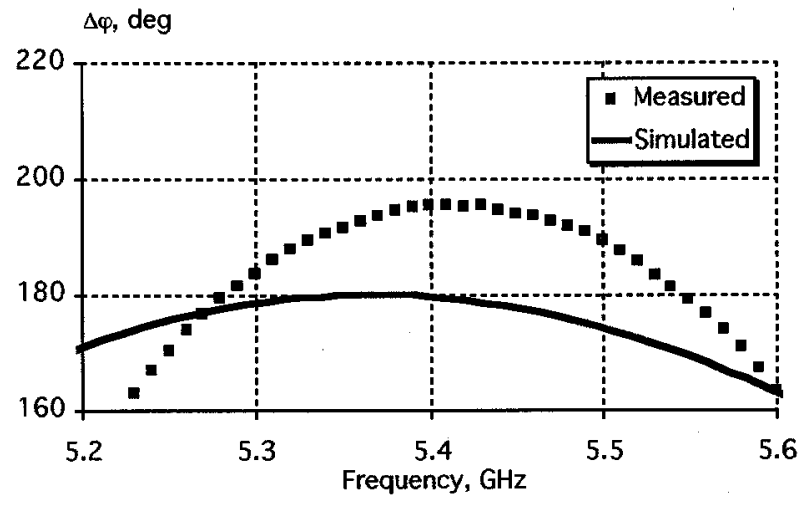

(b)

Fig. 3. (a) Experimental loss-frequency and (b) phase shift-frequency characteristics of $180^{\circ}$ reflection-type phase shifter in comparison with the simulated ones for $\tan \delta=0.06$. In the experiment, the phase shifter was controlled by dc bias voltage $\left(U_{1}=0 \mathrm{~V}\right.$ and $\left.U_{2}=350 \mathrm{~V}\right)$. 
ated figure of merit of the phase shifter is about $180 \mathrm{deg} / \mathrm{dB}$ for $\tan \delta=0.02$ and $70 \mathrm{deg} / \mathrm{dB}$ for $\tan \delta=0.06$.

\section{MEASUREMENTS}

The SMD blocking capacitors were soldered onto the contact pads. dc bias voltage (up to $350 \mathrm{~V}$ ) was applied to the phase shifter through the protecting $1 \mathrm{M} \Omega$ resistor.

Measurements of the phase shifter were done using the microwave coplanar probe station of HP8720 Network Analyzer.

The measured loss of the phase shifter (Fig. 3) is similar to the simulated characteristic of the phase shifter based on the capacitor with $\tan \delta=0.06$. The CQF of the capacitor is 90 and the corresponding figure of merit of the phase shifter is about 60 $\mathrm{deg} / \mathrm{dB}$.

\section{CONCLUSION}

The reflection-type one-bit ferroelectric phase shifter has been designed, fabricated, and tested. The developed theory of the ferroelectric phase shifter allows designing the phase shifters with optimized characteristics. Integration of the strip matching circuit into the coplanar line structure gives a possibility to design the optimized digital phase shifter as an integrated circuit. The cost of such an integrated circuit is much less, than the cost of the FET phase shifter based on GaAs integrated circuit. As compared to the $\mathrm{p}-\mathrm{i}-\mathrm{n}$ diode phase shifter, the ferroelectric phase shifter is preferable due to extremely small power in de control circuits.

\section{REFERENCES}

[1] F. W. Van Keuls, R. R. Romanofsky, N. D. Varaljay, F. A. Miranda, C. L. Canedy, S. Aggarwal, T. Venkatesan, and R. Ramesh, "A Ku-band gold/ $/ \mathrm{Ba}_{x} \mathrm{Sr}_{1-x} \mathrm{TiO}_{3} / \mathrm{LaAlO}_{3} /$ conductor thin-film ferroelectric microstrip line phase shifter for room-temperature communication applications," Microwave Opt. Technol. Lett., vol. 20, pp. 53-56, Jan. 1999.

[2] A. Kozyrev, V. Osadchiy, A. Pavlov, and L. Sengupta, "Application of ferroelectrics in phase shifter design," in IEEE MTT-S 2000 Int. Microwave Symp. Dig., June 2000, pp. 1355-1358.

[3] A. Kozyrev and A. Ivanov, "Ferroelectric films: Nonlinear properties and applications in microwave devices," in IEEE MTT-S 1998 Int. Microwave Symp. Dig., June 1998, pp. 985-988.

[4] S. K. Koul and B. Bhat, Microwave and Millimeter Wave Phase Shifters. Norwood, MA: Artech House, 1991.

[5] I. B. Vendik, O. G. Vendik, E. L. Kollberg, and V. O. Sherman, "Theory of digital phase shifters based on high-Tc superconducting films," IEEE Trans. Microwave Theory Tech., vol. 47, pp. 1553-1562, Aug. 1999.

[6] I. B. Vendik, O. G. Vendik, and E. L. Kollberg, "Criterion for a switching device as a basis of microwave switchable and tunable components," in Proc. 29th Eur. Microwave Conf., vol. 3, Munich, Germany, 1999, pp. 187-190.

[7] - "Commutation quality factor of two-state switchable devices," IEEE Trans. Microwave Theory Tech., vol. 48, pp. 802-808, May 2000. 